\title{
Strangulation de la verge par anneau métallique compliquée d'une lésion urétrale
}

\author{
Kamel CHABCHOUB, Elmostafa LOKMANE, Jihad EL HAJJ, Philippe DANJOU
}

Service d'urologie, Centre Hospitalier Dr Schaffner, Lens

\section{RÉSUMÉ}

Nous rapportons le cas d'un patient âgé de 61 ans admis aux urgences pour un lymphoedème pénien évoluant depuis 3 semaines après la pose volontaire d'un anneau métallique de porte-clés à la base de la verge dans un but d'automutilation. L'ablation de cet anneau a été immédiatement réalisée sous anesthésie locale.

Sept jours plus tard, après chute d'escarre, une fistule urétrale s'est déclarée au niveau péno-scrotal, ce qui a nécessité un drainage vésical par cathéter sus-pubien. II a été décidé de réaliser secondairement la fermeture chirurgicale de la fistule et le recouvrement cutané de la base de la verge après amélioration de l'état local de la plaie.

A travers cette observation et les données de la littérature, nous précisons la prise en charge de cette situation pathologique et des complications associées.

Mots clés : anneaux métalliques, strangulation du pénis, fistule urétrale

\section{INTRODUCTION}

La strangulation de la verge par anneau métallique ou non est rare. Facilement diagnostiquée, elle impose de réfléchir à la manière de retirer l'anneau et à la réparation des dégâts. Ces dégâts sont potentiellement graves en raison des complications urinaires ou sexuelles. Nous rapportons un cas de strangulation pénienne par anneau métallique compliquée d'une lésion urétrale.

\section{OBSERVATION}

Monsieur V. C., âgé de 61 ans, sans antécédents pathologiques particuliers, a été admis aux urgences pour douleur et œdème de la verge évoluant depuis trois semaines. L'examen clinique montrait un double anneau métallique de porte-clés bloqué à la base de la verge et un important lymphœdème d'aval sans signes d'ischémie ni de nécrose pénienne.

Le diamètre et la longueur de la verge étaient respectivement de $5 \mathrm{~cm}$ et de $14 \mathrm{~cm}$. La peau et le dartos en regard de l'anneau étaient profondément lésés et infectés (Figure 1). II n'a été noté ni rétention d'urine ni fistule urétrale. L'ablation de cet anneau a été

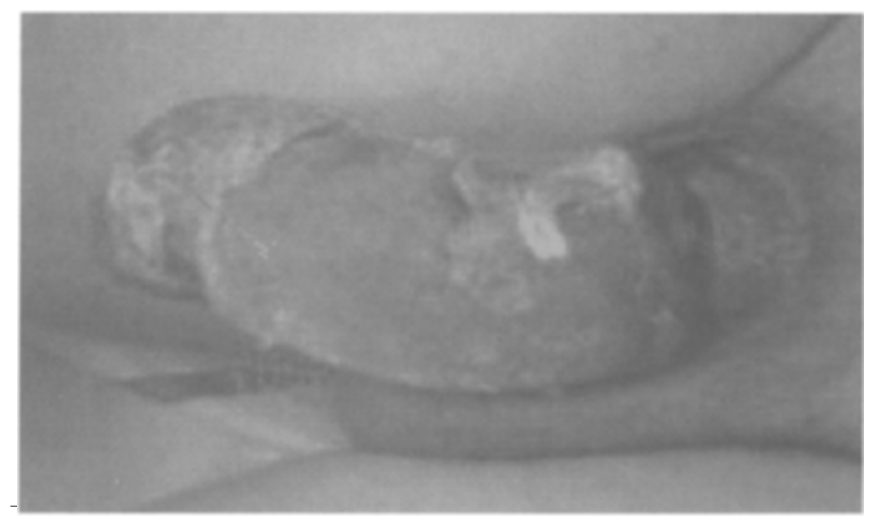

Figure 1 : Important lymphoedème pénien avec hyper kératose cutanée en amont d'un anneau métallique bloqué à la base de la verge. Ulcération cutanée profonde en regard de l'anneau.

Correspondance :

Dr Kamel CHABCHOUB - Senvice Urologie, Centre Hospitalier Dr Schaffner, 99, Route de la Bassée, 62307 Lens Cedex - Telo6 61205003 - Fax 0321691575 Emailk.chabchoub@laposte.net 
immédiatement faite sous anesthésie locale. Le geste a consisté en l'infiltration circonférentielle de $10 \mathrm{ml}$ de lidocaïne $1 \%$ à la base de la verge au voisinage de la zone lésée, suivie par une double section de l'anneau à l'aide d'une pince coupante (pince à broches utilisée en chirurgie orthopédique). A l'ablation de cet anneau les corps caverneux étaient à nu sans lésion spongieuse. La verge a été maintenue en position verticale et un bandage de contention a été mis en place après décapage des lésions cutanées, en vue de faciliter le drainage lymphatique.

Pendant l'entretien psychologique, le patient a exprimé un sentiment de culpabilité face à des problèmes familiaux. II a avoué la nature volontaire de son geste dans un but d'automutilation. II n'a consulté que trois semaines après, devant l'échec d'extraction de l'anneau et l'aggravation de la symptomatologie.

Sept jours après l'ablation de l'anneau, après chute d'escarre, une fistule urétrale s'est déclarée au niveau péno-scrotal (Figure 2), ce qui a nécessité un drainage vésical par cathéter sus-pubien. Au quinzième jour, la verge avait nettement diminué de volume et la peau était plus souple et moins infiltrée (Figure 3). II a été décidé de réaliser une fermeture chirurgicale de la fistule et un recouvrement cutané de la base de la verge après traitement de l'infection de la plaie et amélioration de la qualité de la peau pénienne.

\section{DISCUSSION}

La strangulation de la verge par anneau est rencontrée en particulier, chez des patients psychologiquement déséquilibrés [4]. Le motif de ce geste volontaire est soit l'auto-mutilation [4, 6] soit l'auto-érotisme ou même le désir d'améliorer la performance sexuelle par une rigidité pénienne plus durable [7]. Nombreux sont les dispositifs bloqués à la base de la verge. II peut s'agir d'anneaux métalliques (bague, anneau de rideau, porte-clés ...) ou non métalliques (collet de bouteille plastique ...) [6].

La strangulation du pénis se manifeste précocement par un œdème rendant impossible l'extraction de l'anneau par le patient lui-même, dès les premières heures. Ceci est dû à l'interruption rapide de la circulation veineuse et lymphatique cutanée. L'évolution ultérieure dépendra de la sévérité de l'obstruction.

Pour les anneaux serrés, l'ischémie puis la nécrose s'installent précocement $[4,7]$. Ceci est observé chez des patients jeunes qui cherchent à maintenir une érection prolongée par l'utilisation d'anneaux serrés comprimant toute la verge [7]. Pour les anneaux peu serrés, provoquant une souffrance du revêtement cutané et sous cutané, l'ischémie de la verge est absente. II

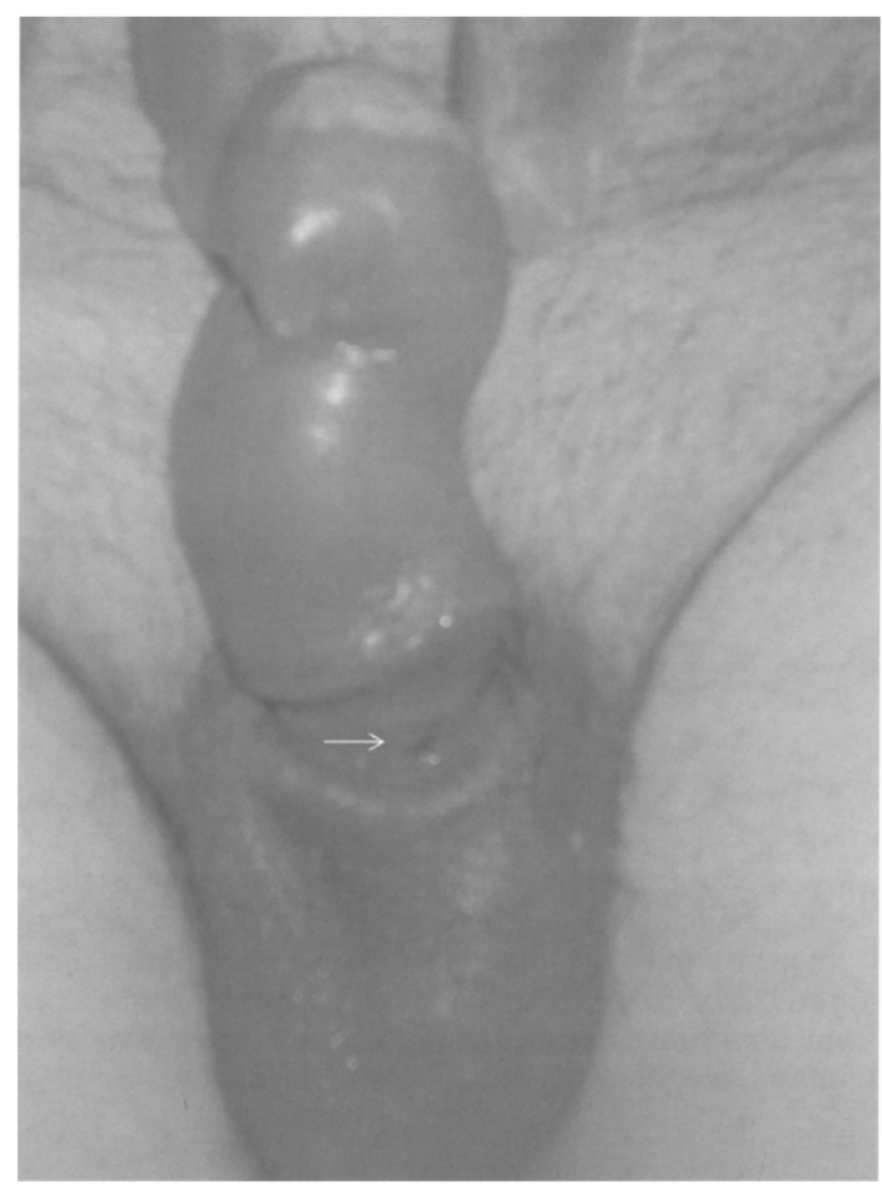

Figure 2 : Septième jour après ablation de l'anneau.

Diminution du volume de la verge et apparition d'une fistule urétrale de $1 \mathrm{~cm}$ de large au niveau péno-scrotal (flèche).

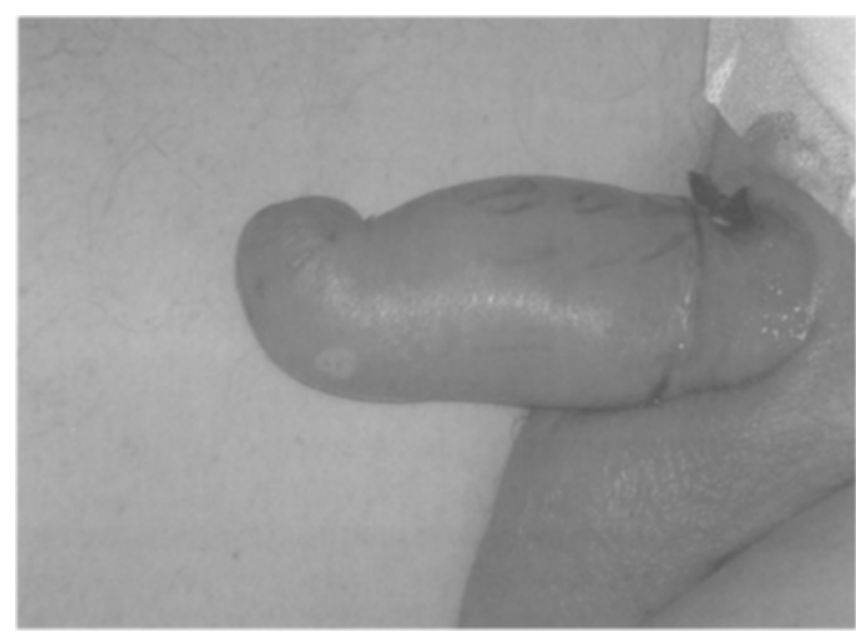

Figure 3 : Quinzième jour après ablation de l'anneau.

Normalisation de l'aspect de la verge. 
s'installe secondairement un lymphoedème d'aggravation progressive au niveau de la peau pénienne, donnant à long terme un aspect cartonné et tardivement éléphantiasique [8]. Notre patient n'a consulté qu'après trois semaines au stade de début du lymphoedème pénien.

Le traitement doit être urgent [5]. La première étape consiste en l'ablation du matériel compressif souvent sous anesthésie locale. La technique utilisée dépend de la dureté et de la forme de l'anneau. Pour les anneaux peu épais et peu larges, la section est généralement facile, faite à l'aide de pinces coupantes. En revanche, ce geste est difficile pour les anneaux durs épais et/ou larges ne pouvant pas être saisies par les pinces coupantes [6]. Dans ces cas, il est recommandé de drainer le sang stagnant dans la verge par incision ou ponction du gland suivie d'un bobinage compressif de la verge par un fil de soie sur lequel est glissé progressivement l'anneau $[3,5,9]$.

La deuxième étape comporte le débridement des berges ischémiques et nécrotiques de la plaie suivi d'un examen minutieux de l'urètre à la recherche d'une fistule notamment en présence d'ulcération profonde [6]. La pose d'un cathéter sus-pubien est nécessaire lorsqu'il existe une fistule urétrale ou une rétention d'urine [1]. L'amputation de la verge s'impose en cas de nécrose pénienne. Elle est réalisée de façon différée de un à huit jours par rapport à l'ablation de l'anneau, dans le but d'attendre une éventuelle récupération $[4,7]$.

Le traitement des lymphoedèmes au stade de début fait appel aux soins cutanés et au drainage lymphatique par bandage de contention [10]. A un stade tardif de sclérose cutanée (éléphantiasis), le traitement consiste en la résection du tissu scléreux suivie d'une plastie du fourreau de la verge [8].

Les lésions urétrales associées sont souvent sales, à berges contuses ou déchiquetées et parfois avec perte de substance, la suture n'est pas possible et ne doit pas être tentée [2]. II faut alors éviter les manœuvres agressives de mobilisation urétrale ou de parage extensif du tissu spongieux, et préférer un drainage par cathéter sus-pubien suivi d'une réparation secondaire de la fistule urétrocutanée lorsque la peau devient de bonne qualité et en l'absence de sepsis [2].

La prise en charge psychologique et sexologique est indispensable chez ces patients, particulièrement chez ceux qui souffrent d'un déséquilibre psychiatrique, et lorsqu'il existe une complication grave, amputation ou éléphantiasis de la verge.

\section{CONCLUSION}

La strangulation de la verge par anneau est diagnostiquée chez des patients psychologiquement et sexuellement déséquilibrés. La nécrose et l'éléphantiasis de la verge sont les complications les plus graves. L'ablation urgente de l'anneau est la première étape thérapeutique. Le traitement ultérieur doit être adapté en fonction des lésions associées et de leur évolution et comporte systématiquement une prise en charge psychosexologique.

\section{RÉFÉRENCES}

1. GONZALEZ-SATUE C., RIERA CANALS L., FONTDEVILA J., ESTRADA J., ARBELAEZ ARANGO S., TRILLA HERRERA E. et al. : Penile and urethral lesion caused by metallic ring. Reconstruction with radial free flap. Actas Urol. Esp., 2001, 25 (10) : 746-749.

2. MANUNTA A., VINCENDEAU S., PATARD J-J., LOBEL B., GUILLÉ F. : Traumatismes récents de l'urètre masculin. Encycl. Méd. Chir. Techniques chirurgicales Urologie, Paris, Elsevier, $2004: 41-330$.

3. MCLAUGHLIN T., COYNER W. : Removal of a strangulating metal bearing from the penis. J. Urol., 1989, $141: 617$.

4. MOUFID K., JOUAL A., DEBBAGH A., BENNANI S., EL MRINI M. : L'automutilation génitale : à propos de 3 cas. Prog. Urol., 2004, 14 : 540-543.

5. NOH J., KANG T.W., HEO T., KWON D.D., PARK K., RYU S.B. : Penile strangulation treated with the modified string method. Urology, 2004, $64: 591$.

6. PERABO F.G., STEINER G., ALBERS P., MULLER S.C. : Treatment of penile strangulation caused by constricting devices. Urology, 2002, $59: 137$.

7. OSMAN M., AL KAD| H., AL HAFIR. : Gangrene of the penis due to strangulation by a metallic ring. Scand. J. Urol. Nephrol., 1996, $30: 77-78$.

8. TANABE N., MUYAM., ISONOKAMI M., KOZUKAT., HONDA T., OHTANI $H$. : Lymphedema due to chronic penile strangulation: a case report. J. Dermatol., 1996, 23 : 648-651.

9. VAHASARJA V.J., HELLSTROM P.A., SERLO W., KONTTURI M.J. : Treatment of penile incarceration by the string method: 2 case reports. J. Urol., 1993, $149: 372-373$.

10. VAILLANT L., GIRONET N., BAULIEU F. : Lymphoedèmes des membres. Encycl. Méd. Chir. Paris, Elsevier, Dermatologie, 98-575-A-10, $2002: 10$.

Manuscrit reçu : août 2006 ; accepté septembre 2006. 


\begin{abstract}
Penile strangulation by metal ring complicated by urethral lesion

Kamel CHABCHOUB, Elmostafa LOKMANE, Jihad EL HAJJ, Philippe DANJOU
\end{abstract}

Penile incarceration is uncommon. The diagnosis is obvious, but extraction of the constrictive ring and repair of the damage can be challenging.

The authors present the case of a 61-year-old man wh presented with pain and swelling of the penis 3 weeks after applying a metal ring (double key rings) at the base of penis for the purposes of self-mutilation. The key ring was removed immediately under local anaesthesia. One week later, a urethral fistula was observed, requiring suprapubic cystostomy. It was decided to perform secondary surgical closure of the fistula and reconstruction of the skin of the base of the penis after treatment of local sepsis.

Penile incarceration should be considered to be an emergency, as the more rapidly the constrictive object is removed, the lower the risk of complications secondary to penile devascularization, urinary retention and urethral damage. The authors review the literature concerning this unusual injury and its management.

Keys word : metal rings, penile strangulation, urethral fistula 